\title{
Robotic therapy for the hemiplegic shoulder pain: a pilot study
}

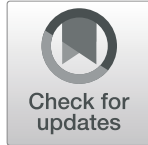

\author{
Ruthber Rodríguez Serrezuela ${ }^{1 *}$, Mauricio Torres Quezada², Marcia Hernández Zayas³, \\ Arquímedes Montoya Pedrón ${ }^{3}$, Daily Milanés Hermosilla² and Roberto Sagaró Zamora ${ }^{2}$
}

\begin{abstract}
Backgrounds: Exoskeletons development arises with a leading role in neurorehabilitation technologies; however, very few prototypes for upper limbs have been tested, contrasted and duly certified in terms of their effectiveness in clinical environments in order to incorporate into the health system. The purpose of this pilot study was to determine if robotic therapy of Hemiplegic Shoulder Pain (HSP) could lead to functional improvement in terms of diminishing of pain, spasticity, subluxation, the increasing of tone and muscle strength, and the satisfaction degree.

Methods: An experimental study was conducted in 16 patients with painful shoulder post- ischemic stroke in two experimental groups: conventional and robotic therapy. At different stages of its evolution, the robotic therapy effectiveness applied with anti-gravitational movements was evaluated. Clinical trial was developed at the Physical Medicine and Rehabilitation Department of the Surgical Clinical Hospital "Dr. Juan Bruno Zayas Alfonso" in Santiago de Cuba, from September 2016 - March 2018. Among other variables: the presence of humeral scapular subluxation (HSS), pain, spasticity, mobility, tone and muscle strength, and the satisfaction degree were recorded. Results with 95\% reliability were compared between admission and third months of treatment. The Mann-Whitney U-Test, ChiSquare and Fisher's Exact Tests were used as comparison criteria.
\end{abstract}

Results: Robotic therapy positively influenced in the decrease and annulment of pain and the spasticity degree, reaching a range increase of joint movement and the improvement of muscle tone.

Keywords: Stroke, Neurorehabilitation, Robotic therapy, Hemiplegic shoulder pain

\section{Introduction}

The World Health Organization (WHO) consider neurorehabilitation as an active process where some individuals, with some injury or neurological disease, could achieve the most optimal integral recovery in order to integrate their activities with the environment in the most appropriate approach. Neurorehabilitation technologies have undergone important changes in the last years due to an exponential increase (according to the health systems data) of cerebrovascular accidents (CVA)

\footnotetext{
* Correspondence: ruthber.rodriguez@corhuila.edu.co

${ }^{1}$ University Corporation of Hulia, Nevia, Huila, Colombia

Full list of author information is available at the end of the article
}

as the third leading cause of death in the world and high rate of disability.

In Cuba, the disease incidence is estimated at 22,000 cases per year, where about 9725 patients die $[1,2]$. It is expected that 70 new strokes occur in the country per day. In $50 \div 70 \%$ of survivors remain sequels, and about a third are unable to fend for themselves, calculating that approximately $75 \%$ lose their ability to return to work [3]. This entity represents the fourth national cause of potentially lost life years, with around 11 years for a rate of 86.9 per 1000 inhabitants [4].

Exoskeletons with rehabilitation purposes have reached a great media diffusion and their development occupies a significant number of research groups in the world. However, although more than 100 prototypes have been developed in

C The Author(s). 2020 Open Access This article is licensed under a Creative Commons Attribution 4.0 International License, which permits use, sharing, adaptation, distribution and reproduction in any medium or format, as long as you give appropriate credit to the original author(s) and the source, provide a link to the Creative Commons licence, and indicate if changes were made. The images or other third party material in this article are included in the article's Creative Commons licence, unless indicated otherwise in a credit line to the material. If material is not included in the article's Creative Commons licence and your intended use is not permitted by statutory regulation or exceeds the permitted use, you will need to obtain permission directly from the copyright holder. To view a copy of this licence, visit http://creativecommons.org/licenses/by/4.0/ The Creative Commons Public Domain Dedication waiver (http://creativecommons.org/publicdomain/zero/1.0/) applies to the data made available in this article, unless otherwise stated in a credit line to the data. 
recent years, only 10 have been clinically tested and they are commercial. In clinical rehabilitation tasks, only $2 \%$ are used [5-7]. Nowadays there is not enough clinical evidence, nevertheless some reports include the possibility to improve the functional result after the CVA [8-13].

In the last two decades, some important meta-analyses, including a number of randomized controlled trials, have been performed to investigate the therapeutic effects of upper limb robotic rehabilitation [14-16]. These studies have shown that robotic therapy either produce improvements in the motor control and muscle strength of the paretic arm without side effects or is more effective than conventional therapy in improving upper limb motor function recovery, especially in chronic stroke patients.

The main topics discussed in these investigations are linked to the following aspects: improving motor recovery in distal and proximal joints, the effect of robotic therapy in chronic patients and those design parameters such as power/weight ratio actuators, DOF, accurate, compactness, greater range of motion, safe operation, reliability in all operations, relatively low complexity and low engineering and construction cost, simple fitting and removal, comfort in wearing, and easy maintenance. Some research has deeply studied the combination with other neurorehabilitation technologies (BCI/BMI, FES and $\mathrm{tDCs}$ ) in recent years.

Although there are well-documented research on the use of exoskeletons in the rehabilitation of the upper limb post-stroke patients, only a few reports have been focused to the treatment of the painful shoulder of the hemiplegic patient using robotic therapy $[8,12,13]$.

Hemiplegic Shoulder Pain (HSP) after CVA is a common and debilitating complication. Its incidence varies between $16 \%$, in patients with flaccid paresis and up to $84 \%$ in severe paralysis with significant development of spasticity. It may occur in the acute phase (in the first 2 weeks) but more often after two or 3 months, generating greater difficulty in mobilizing the limb, greater disability in transfers, trunk balance and carrying out daily living activities, hindering functional recovery of the upper extremity.

Researchers at Robotics Research Laboratory from the Feinstein Institute for Medical Research [8] conducted an uncontrolled pilot trial in 18 chronic hemiplegic patients using an end-effector exoskeleton. The patients presented glenohumeral subluxation as the only etiology of the painful shoulder, and spasticity was reported in an unspecified number of them. It reported a significant decrease in subluxation, spasticity and an increase in muscle tone as the main results. In their study, however, it is not possible to infer the degree of spasticity of patients during admission and no relationship is established between subluxation and pain, and how the latter changed during treatment. Although an increase in muscle tone is established because of therapeutic treatment, the ranges of active mobility (ROM) are not reported during the beginning and the end of the training.

In a randomized controlled trial with 19 patients (eight who received robotic therapy), after $1 \div 8$ weeks poststroke, researchers from the University of Guelph [12] analyzed the influence of robotic therapy in the rehabilitation of the upper limb of hemiplegic patients (three patients with flaccid limb) and hemiparetic. No subluxated patients were reported in this study and shoulder pain (light or medium) is attributed to other lesions of the musculoskeletal system. The main results indicate a significant motor recovery in the arm and hand, but no significant changes in pain are reported, and muscle spasticity is not included in the study.

Researchers at Wonkwang University [13] have conducted a study of the effectiveness of an exoskeleton prototype for the rehabilitation of painful shoulder in the hemiplegic patient. The controlled study includes 36 patients divided into two groups, experimental (11 subluxated patients and 14 with spasticity) and control (11 subluxated and 13 spastic patients). The most important results were the decrease in pain between admission and discharge in the robotic therapy group, but not in the control group. The device, however, only incorporates improvements in shoulder abduction, not being successful for internal and external rotations and shoulder flexion/extension. The levels of improvement in patients' subluxation of the shoulder and the evolution of spasticity during discharge were not reported.

All these results show the inconsistencies of conventional therapies and the possibilities of using robotic exoskeletons. However, these studies have, as a limitation, not to consider the multifactorial genesis of the HSP syndrome. Therefore, the main purpose of our study is to analyze the influence of changes in abnormal muscle tone like flaccid limb, spasticity (mainly in subscapularis and pectoralis muscles) on the evolution of pain as a result of three controlled therapeutic shoulder exercises performed with robotic therapy.

\section{Methods}

An experimental study in patients who survived ischemic stroke with lesion corresponding to the territory irrigated by the middle cerebral artery, who presented HSP syndrome, in order to evaluate the effectiveness of robotic therapy with anti-gravitational movements was performed. The total number of patients with motor deficit who met the selection criteria was included. If the individuals met the inclusion criteria and gave informed consent, they were assessed, then, by an occupational therapist who was blinded to the study and not directly involved with patient care. 
Voluntariness, age over 40 years, flaccid and spastic stages (up to grade 2, Modified Ashworth Scale, MAS) of the hemiplegic shoulder and first episode of cerebrovascular disease, body weight and height up to $100 \mathrm{~kg}$ and $175 \mathrm{~cm}$ respectively, were parameters considered for the inclusion. Some features were excluded: cognitive, sensory, sensitive and communication deficits that made it difficult to understand and follow instructions; pain, motor deficit and/or previous diagnosis of musculoskeletal shoulder disorder, as well as the clinical conditions that contraindicate the rehabilitation treatment. The following were considered as output criteria: voluntary abandonment, appearance of clinical complications that interfered with the study and/or rehabilitation treatment, recurrence of stroke, absence of 3 treatment sessions, and death.

Two stages of evolution were observed in the affected upper extremity: flaccid (flaccidity, tendon reflex, hypotonic, the patient cannot initiate any specific motor function) and spastic (where hypertonia, glenohumeral subluxation, depression, scapula retraction, adduction and internal rotation of the humerus can be found). This spastic phase can be complete, initiating a total synergy, or partial and incomplete. The variables related to the clinical-epidemiological characteristics collected were evolution time, affected hemibody and paralysis degree: hemiparesis/hemiplegia. According to the radiological criteria established by Prevost and Arsenault humeral scapular subluxation (HSS) was considered as Absent, Moderate $(<7 \mathrm{~mm})$ or Severe $(>7 \mathrm{~mm})$ [12].

\section{Experimental protocol}

Patients with anthropometric characteristics $168 \pm 12 \mathrm{~cm}$ of height, and $75.7 \pm 15.4 \mathrm{~kg}$ of weight concluded 3 months of therapy in the grounded robotic platform of 4 freedom degrees developed by the Mechanical and Industrial Engineering Department [17, 18] (Fig. 1). Individuals randomized to the experimental group completed at a rate of 1 effective hour per day, 5 weekly sessions with anti-gravitational movements. The protocol to follow and the experimental procedures, including all the rehabilitation routines and security rules were explained to the participants. No additional visual or audio-visual feedback were supplied.

The therapy was performed in a sitting position with neutral or starting position of the upper extremity. The movement routines selected for the study were: flexion/ extension of shoulder and elbow, external and internal rotation and abduction of the upper limb, and prono/supination of wrist. The abduction movement is achieved using shoulder flexion/extension actuator and the counter clock rotation of a patient special-chair design. This particular design (set exoskeleton-chair) allows aligning the rotational axis of the actuator that ensure the flexion/extension movements of the shoulder, with the anatomical axis of the patient for executing abduction/adduction movements. In this way the therapeutic movements of flexion/extension, internal/external rotation and abduction/adduction are performed during the shoulder rehabilitation.

No other kinds of therapy (including thermotherapy, used in those patients of control groups) were programmed for the experimental group.

Exoskeleton is able to permit a physiological range of mobility for selected movements, and the displacement angles were controlled by physiotherapist trough an interface designed for this purpose. Initial values for every patient in each session of the selected routines were fixed considering the maximum pain-tolerable range of motion of the shoulder joint.

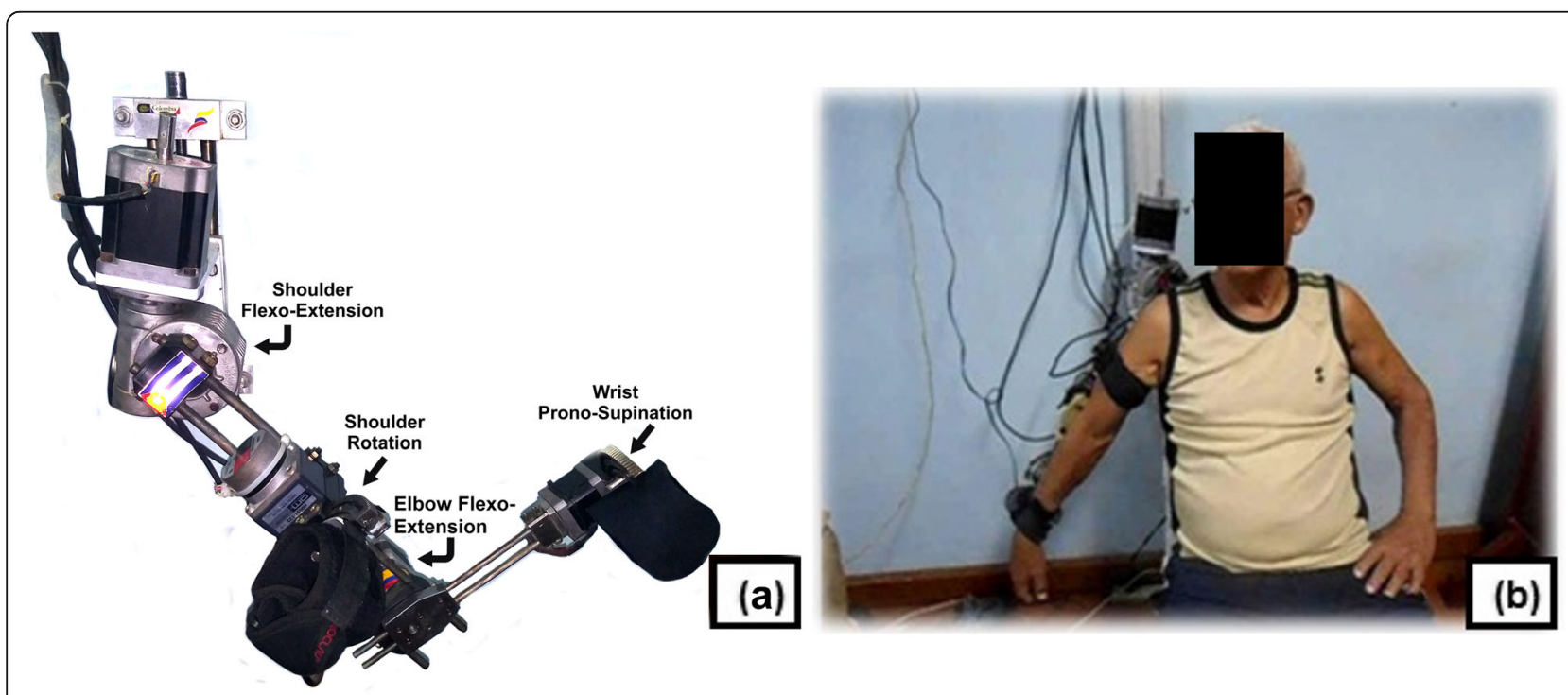

Fig. 1 a Exoskeleton for upper limb rehabilitation. b Patient during abduction training in robotic therapy 


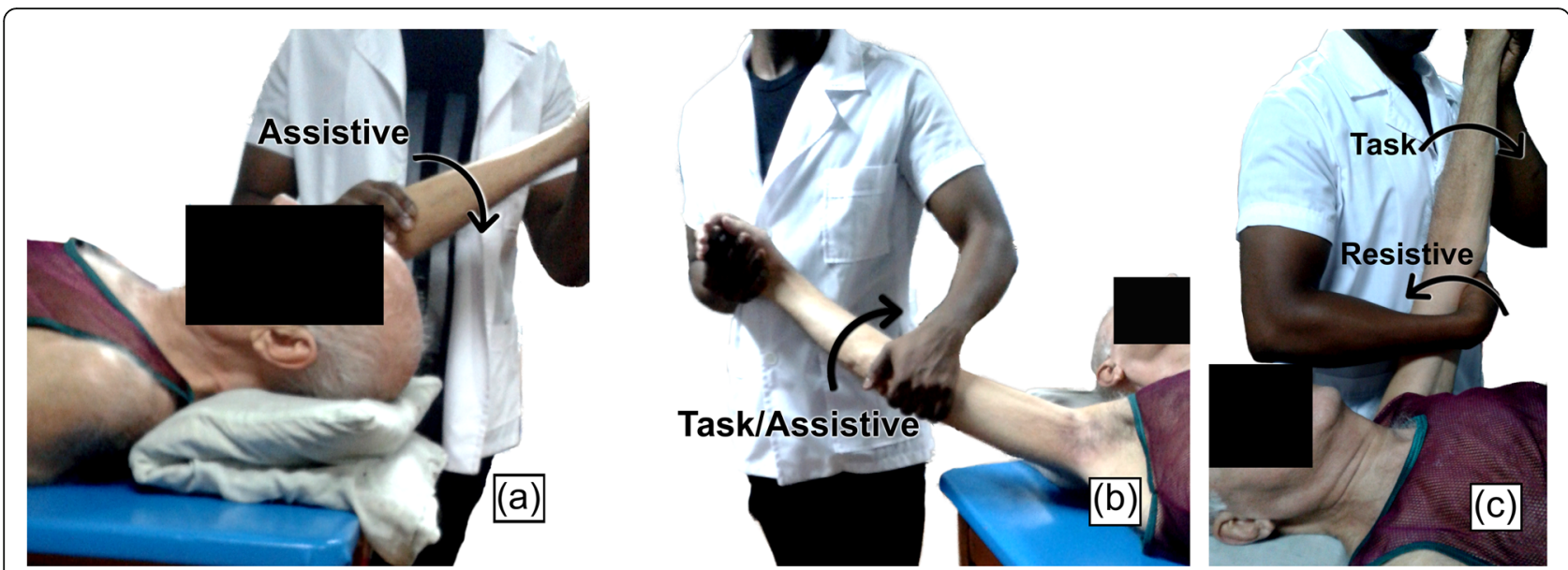

Fig. 2 Exercises in conventional therapy. a Passive exercise in shoulder flexion assisted by the physiotherapist. b Active abduction exercise assisted by the physiotherapist. c Active-resistive exercise during the flexion/extension movement of the shoulder

Patients of conventional therapy group $(174 \pm 6.7 \mathrm{~cm}$ of height, $77 \pm 13.1 \mathrm{~kg}$ of weight) first received superficial thermotherapy [19-22]. The expected therapeutic effects of heat are analgesic, antispasmodic, anti-inflammatory, and the preparation for the exercise. Infrared heat was use for $15 \mathrm{~min}$ at a distance of $70 \div 80 \mathrm{~cm}$ according to the affected shoulder and later lymphatic massage and kinesitherapy that included the same movements practiced by robotic therapy group. The kinesitherapy scheme (one effective hour) consisted of the combination of exercises, guided in movement progression and in all its planes. The control group (as well as the robotic therapy group) included hemiplegic and hemiparetic patients, and for this reasons the set of movements involved manual exercises based on Kabat approaches (Fig. 2):

Assisted Passives by the physiotherapist, for those patients unable to execute the indicated movement.

Self-assisted passives by the patient himself

Assisted actives by the physiotherapist, for those patients with certain mobility but unable to complete the movement.

Resisted (actives), for those patients capable of completing the movement, and who, as part of their muscular recovery during the performance of the movements receive a resistive force from the physiotherapist.

These exercises incorporated the nature of the movements selected for the study (i.e. shoulder flexion/extension, internal and external rotation, abduction) and were indicated in correspondence with muscle tone, assessed for all patients according to their classification in the MRC scale and their evolution.

In both experimental and control group, each set of repetitive movement will be divided into 3 time sessions with 10 repetitions each.

\section{Outcome measures}

Omalgia assessment: instruments were applied, which allowed weighting the clinical variables indicating their evolution (pain and mobility, muscle tone and strength, active joint movement range, spasticity and satisfaction degree). All of them were recorded at the beginning, every 10 sessions of treatment, with a monthly summary and after 3 months of robotic therapy. Radiological and neurophysiological studies were also performed at the beginning and the end of the treatment.

Pain assessment: The severity degree of the painful shoulder was defined $[7,11]$ in four grades. Severe (0), pain and functional limitation while rest with all movements limited. Moderate (1), pain that intensifies with movement and very light while resting, the movements are painful, but there is no limitation in the joint range. Light (2) without pain at rest, only occurs with rapid movements or under active mobilization and almost normal (3), where only pain appears or limitation to active movements resisted.

Spasticity Degree: it was classified according to the well-known numerical Scale of Ashworth Modified $(0 \div 4)[7,11,23]$ that establishes, in ascending order, from normal muscle tone to extreme hypertonia, due to hyperactivity of the mitotic reflex arc.

Muscular testing: Based on the application of the Medical Research Council scale (MRC) [16, 24], that establishes different degrees of muscular strength $(0 \div 5)$ and that in ascending level conceptualizes from the absence of muscular contraction (grade 0) up to normal muscle strength (grade 5).

Articular assessment of motion range: Measurement by goniometry was subdivided into three categories, nonfunctional shoulder: active flexion less than $60^{\circ}$ and abduction less than $40^{\circ}$; with improvement: when actively 
performing shoulder flexion at $60^{\circ}$ and $40^{\circ}$ in abduction, and, functional shoulder: with $\geq 90^{\circ}$ of flexion and $\geq 75^{\circ}$ or more of active abduction [12].

Degree of subjective general satisfaction: Likert scale of 10 points ranging from 1 (Nothing) to 10 (A lot) allowing to define the perception of satisfaction level with the improvement degree and its acceptance in relation to the exoskeleton [12].

The results were statistically processed with a 95\% reliability when compared between the admission and 3rd month of treatment in order to evaluate its significance level of improvement. The professional package Statgraphics Centurion XV.1 was used and included as comparison criteria, the Mann-Whitney U-Test, Chi-Square and Fisher's Exact Tests. $P$ values of $\leq 0.05$ were considered statistically significant.

\section{Patients}

It was included as a universe for the total number of patients with motor deficit (hemiplegia/hemiparesis) of ischemic cause with lesion corresponding to the territory of the middle cerebral artery, which presented painful shoulder in flaccid or spastic stages and which complied with the selection criteria. In a group of 18 patients evaluated, two patients did not meet the inclusion criteria.

The 16 patients selected for the study were randomly divided into two groups: one of robotic therapy or experimental group, and one of conventional therapy or control group. A physiotherapist unrelated to the study randomized the participants into one of two groups using a random number Table. A research technician from Universidad de Oriente collected the biomechanical and progress data from the robotic system at admission and discharge. An occupational therapist blinded to patient allocation administered the outcome measures at admission and discharge.

The clinical protocol was applied to eight patients, all with medical diagnosis of HSP, in the pilot study five men and three women, with an average age of $63.4 \pm$ 15.9 years were included. The control group consisted of 4 men and 4 women, with an average age of $64 \pm 19.7$.
Tables 1 and 2 show the general characteristics of the patients included in both study groups.

Tables 3 and 4 show the evaluation indices during admission for each patient in both groups according to the declared measurement instruments. As it can be observed, both groups of patients respond heterogeneously to the different etiologies of the painful shoulder, spasticity degree, muscle tone and severity of the painful shoulder.

In the experimental group, as a painful shoulder etiology, one of the patients presented severe humeral scapular subluxation (HSS, (measured by a single examiner who was not aware of the point of the study)) while in the rest, the existence of the painful shoulder without subluxation was confirmed (SHSS, neurological etiology or other osteomioarticular causes). On the other hand, in the conventional therapy group, one of the patients showed severe scapula-humeral subluxation while in the rest the existence of the painful shoulder without subluxation was confirmed. Thus in the $88 \%$ of patients involved in the study, HSS was absent (by clinical and radiological test), it was confirmed that this etiology was not the main cause of HSP in both groups concerning to this study.

\section{Results}

Tables 5 and 6 show comparatively the main indicators obtained as a result of the application of both therapies in the pilot study.

It can be seen after 3 months of robotic therapy, patients experienced a significant recovery in relation to the control group.

The results obtained from the different indicators, it is deductible that the patients who received robotic rehabilitation exhibited higher rates of motor recovery. From Tables 5 and 6 it is possible to establish the comparison between both groups, in such terms. It should be noted that in relation to the treatment of spasticity, robotic therapy was more consistent in its reduction, with a great influence in the range of active joint mobility and muscle tone increase.

An important result is that patients in this group experienced a pain decrease until its disappearance in just 20

Table 1 General characteristics of the patients involved in the study. Robotic therapy group

\begin{tabular}{lllll}
\hline Patient & Gender & Age & Evolution from CVA (months) & Affected hemisphere \\
\hline S1 & M & 57 & 7 & left \\
S2 & M & 71 & 1 & right \\
S3 & F & 57 & 4 & left \\
S4 & M & 64 & 2 & left \\
S5 & F & 64 & 14 & left \\
S6 & M & 68 & 14 & right \\
S7 & M & 66 & 4 & left \\
S8 & F & 76 & 4 & left \\
\hline
\end{tabular}


Table 2 General characteristics of the patients involved in the study. Control group

\begin{tabular}{lllll}
\hline Patient & Gender & Age & Evolution from CVA (months) & Affected hemisphere \\
\hline C1 & F & 77 & 3 & right \\
C2 & F & 67 & 4 & left \\
C3 & F & 35 & 4 & right \\
C4 & M & 65 & 6 & right \\
C5 & F & 68 & 3 & left \\
C6 & M & 71 & 6 & right \\
C7 & M & 62 & 4 & left \\
C8 & M & 69 & 4 & left \\
\hline
\end{tabular}

sessions and that undoubtedly favors a better balance of all rehabilitation indicators. Other important improvements with the use of robotic therapy were obtained in muscular tone and the increase of ROM. Figure 3 (box plot) and Table 7 show comparatively the range of joint mobility for both groups during admission and after 3 months of treatment for all therapeutical movements.

\section{Discussion}

It can be seen in Table 1 that the patients in experimental group have an average of 6 months after the stroke (three of them are chronic patients, who entered late to the robotic therapy) and 4 months for the conventional therapy group (Table 2). Considering the results of robotic rehabilitation, it can be said that its application is quite promising in patients with a few weeks of poststroke evolution and immobilization of the affected limb. However, it is possible to obtain satisfactory results in chronic patients, whose prognosis for rehabilitation are unfavorable,, as mentioned above, it is one of the most discussed topics related to the application of robotic therapy.

The robotic therapy showed in this study is very effective in the pain treatment if it is compared with the results obtained with the conventional therapy. The authors consider this result linked with the efficacy in the treatment of spasticity and musculoskeletal alterations as the most involved elements in the genesis of HSP [24-29].

In all patients of robotic therapy group, the pain disappeared between $22.5 \pm 4.5$ treatment sessions, result that remained during the following evaluations, while for patients with conventional therapy the pain ceases after $63.7 \pm 19.7$ sessions. One patients of this last group remained at discharge with persisted pain $[p<0.0012$ between both groups]. This result has a great importance in this study since the HSP reduces participation in functional activities and in the rehabilitation process. For conventional treatments, after hospital discharge it predicts a poor functional recovery of the arm, a longer duration of admission and the percentage of patients who are discharged to their home is lower [24, 30-32].

Although there were few analyzed cases, another interesting result, was related to subluxation as one of the etiologies of the painful shoulder of the hemiplegic patient. Figure 4 shows comparatively the subluxation degree of the patient treated with conventional therapy exercises after 3 months. It should be noted that

Table 3 Evaluative indices of patients in the conventional therapy group during admission

\begin{tabular}{llllll}
\hline Patient & ESS $^{1}(\mathrm{~mm})$ & MAS $^{2}$ & MRC $^{3}$ & Painful shoulder severity $^{4}$ & AJM $^{5}$ \\
\hline C1 & - & 2 & 0 & 2 & Not Functional \\
C2 & - & 2 & 1 & 1 & Not functional \\
C3 & $20 \pm 0,4$ & 2 & 0 & Not functional \\
C4 & - & 3 & 2 & 0 & Not functional \\
C5 & - & 1 & 1 & 1 & Not functional \\
C6 & - & 2 & 0 & 1 & Not Functional \\
C7 & - & 1 & 1 & 1 & Not functional \\
C8 & - & 2 & 1 & 1 & Not functional \\
\hline
\end{tabular}

Where:

${ }^{1}$ Evaluation of shoulder stability as measured in $\mathrm{mm}$. (single examiner, blinded to design)

${ }^{2}$ Modified Ashworth Scale

${ }^{3}$ Muscular evaluation according to the scale of the Medical Research Council

${ }^{4}$ Degree of Painful shoulder severity

${ }^{5}$ Degree of functionality of active joint movement according to [12] 
Table 4 Evaluative indices of patients in the robotic therapy group during admission

\begin{tabular}{llllll}
\hline Patient & ESS $^{1}(\mathrm{~mm})$ & $\mathrm{MAS}^{2}$ & $\mathrm{MRC}^{3}$ & Painful shoulder severity $^{4}$ & $\mathrm{AJM}^{5}$ \\
\hline S1 & - & 1 & 2 & 2 & Functional \\
S2 & - & Flaccid & 0 & 1 & Not Functional \\
S3 & - & 0 & 1 & 0 & Not Functional \\
S4 & - & 2 & 1 & 1 & Not Functional \\
S5 & $25 \pm 0,5$ & 1 & 2 & Not Functional \\
S6 & - & 1 & 2 & 2 & Not Functional \\
S7 & - & 2 & 3 & 1 & Not Functional \\
S8 & - & 0 & 2 & 1 & Not Functional \\
\hline
\end{tabular}

Where:

${ }^{1}$ Evaluation of shoulder stability as measured in $\mathrm{mm}$. (single examiner, blinded to design)

${ }^{2}$ Modified Ashworth Scale

${ }^{3}$ Muscular evaluation according to the scale of the Medical Research Council

${ }^{4}$ Degree of Painful shoulder severity

${ }^{5}$ Degree of functionality of active joint movement according to [12]

conventional therapy did not cause favorable changes in the subluxation magnitude.

On the other hand, robotic therapy caused a decrease in the magnitude of subluxated shoulder of $10 \mathrm{~mm}$ after 3 months (Fig. 5).

This patient (S5), arrived to admission in a hemiparetic condition, with a time of evolution of 14 months after the stroke, spasticity degree MAS = 1and a large scapulahumeral subluxation. This is a clear example of how much the HSP delays the rehabilitation of the patient, given that 14 months before entering the study group she showed a very discreet rehabilitation degree. The results obtained in this chronic patient denoted the advantages of robotic rehabilitation even in these cases.

Regarding the treatment of spasticity, the results of the conventional treatment were discrete, which meant that there was an improvement in two of the patients, precisely these two patients, showing an enhancement in the treatment of their spasticity, (moderate to mild hypertonia), they presented successively a recovery of muscle tone and range of mobility. The rest kept their level of spasticity, and one of them worsened $(p<0.45)$.

The systematic anti-gravity training in the exoskeleton caused, however, a reduction and control of spasticity in all cases (four as normal and four with light level, $p<$ $0.02)$. Between both groups MAS scores improved significantly $(p<0.003)$.

This result suggests the importance of controlling and reducing spasticity as the main cause that develops the pattern of pain in the shoulder. Precisely muscle spasticity has been associated with immobility to post CVA, and this study suggests how to treat it with a combination of precise and controlled movements that gradually gain intensity. In this way, it is possible to control the pain and achieve a greater motor recovery evidenced with an increase in muscle tone and ROM.

Furthermore, it is significant, the motor rehabilitation in the flaccid patient (whose prognosis is always

Table 5 Evaluative indices after 3 months of conventional therapy

\begin{tabular}{lllllll}
\hline Patient & ESS $^{1}(\mathrm{~mm})$ & MAS $^{2}$ & MRC $^{3}$ & Painful shoulder severity $^{4}$ & AJM $^{5}$ & Satisfaction Degree $^{6}$ \\
\hline C1 & - & 1 & 2 & No (2 months) & Not functional & 5 \\
C2 & - & 2 & 2 & No (3 months) & Functional & 6 \\
C3 & $20 \pm 0,4$ & 1 & 3 & No (2 months) & Not functional & 5 \\
C4 & - & 3 & 2 & 1 & Not functional & 7 \\
C5 & - & 1 & 2 & No (2 months) & Not functional & 5 \\
C6 & - & 2 & 2 & No (3 months) & Not functional & 6 \\
C7 & - & 1 & 3 & No (2 months) & Not functional & 5 \\
C8 & - & 2 & 2 & No (2 months) & & \\
\hline
\end{tabular}

Where:

${ }^{1}$ Evaluation of shoulder stability as measured in $\mathrm{mm}$. (single examiner, blinded to design)

${ }^{2}$ Modified Ashworth Scale

${ }^{3}$ Muscular evaluation according to the scale of the Medical Research Council

${ }^{4}$ Degree of Painful shoulder severity

${ }^{5}$ Degree of functionality of active joint movement according to [12]

${ }^{6}$ Satisfaction degree of Likert scale 
Table 6 Evaluative indices after 3 months of robotic therapy

\begin{tabular}{lllllll}
\hline Patient & ESS $^{1}(\mathrm{~mm})$ & MAS $^{2}$ & MRC $^{3}$ & Painful shoulder severity $^{4}$ & AJM $^{5}$ & Satisfaction Degree $^{6}$ \\
\hline S1 & - & 0 & $4^{+}$ & No (20 sessions) & Functional & 10 \\
S2 & - & 0 & $4^{+}$ & No (20 sessions) & Functional & 10 \\
S3 & - & 0 & $4^{+}$ & No (20 sessions) & Functional & 10 \\
S4 & - & 1 & 3 & No (1 month) & Functional & 9 \\
S5 & $15 \pm 0,3$ & 1 & 2 & No (20 sessions) & Functional & 9 \\
S6 & - & 1 & 4 & No (20 sessions) & Functional & 10 \\
S7 & - & 1 & 4 & No (1 month) & Functional & 9 \\
S8 & - & 0 & 4 & No (20 sessions) & Functional & 10 \\
\hline
\end{tabular}

Where:

${ }^{1}$ Evaluation of shoulder stability as measured in $\mathrm{mm}$. (single examiner, blinded to design)

${ }^{2}$ Modified Ashworth Scale

${ }^{3}$ Muscular evaluation according to the scale of the Medical Research Council

${ }^{4}$ Degree of Painful shoulder severity

${ }^{5}$ Degree of functionality of active joint movement according to [12]

${ }^{6}$ Satisfaction degree of Likert scale

unfavorable and generally continues until develops spasticity). This patient who experienced an important recovery did not develop spasticity. The same happened with those patients with normal muscle tone during admission.

Other important results are related with the improvement of the range of articular movement (ROM) and muscular tone.

Ranges of joint mobility below those functional, spasticity and absence of muscle tone characterized both groups during admission. As it is known, in the stroke population involved in this kind of experimental studies, there are differences between in general characteristics according to spasticity degree, muscular strength and joint mobility. In this heterogeneity groups exoskeletons can be more helpful and appropriate treatment option for a customized therapy.

In this sense, from Table 7 it can be concluded that the robotic therapy group presented a greater heterogeneity than the control group in ranges of active movement. For example patient S1 (with functional shoulder), who also presented during admission mild pain and hypertonia, $\mathrm{MRC}=2$ and a favorable prognosis of rehabilitation, and patients S6 y S7 with functional values

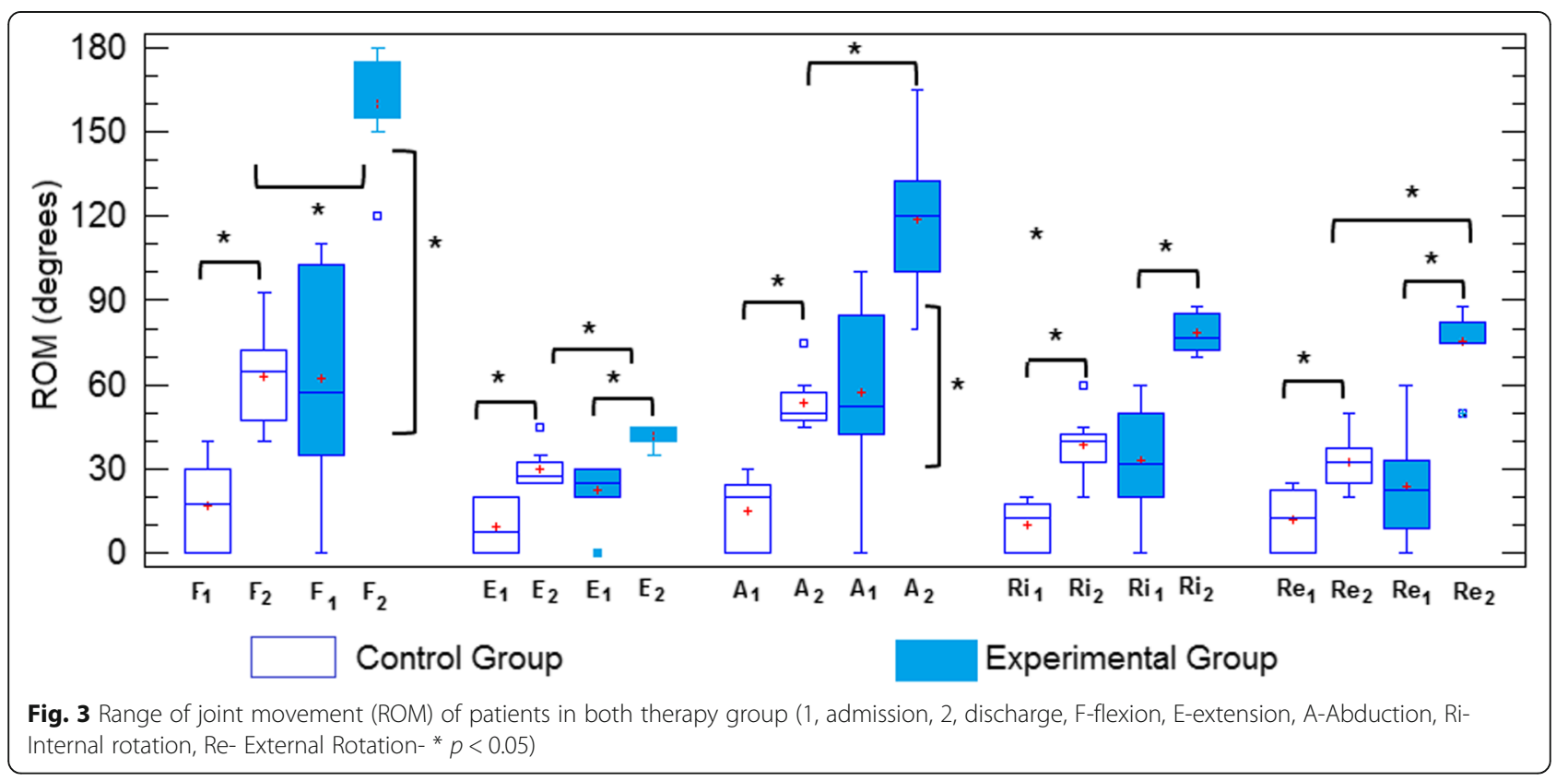


Table 7 Changes in outcome measures (ROM) observed in experimental and control groups after the intervention

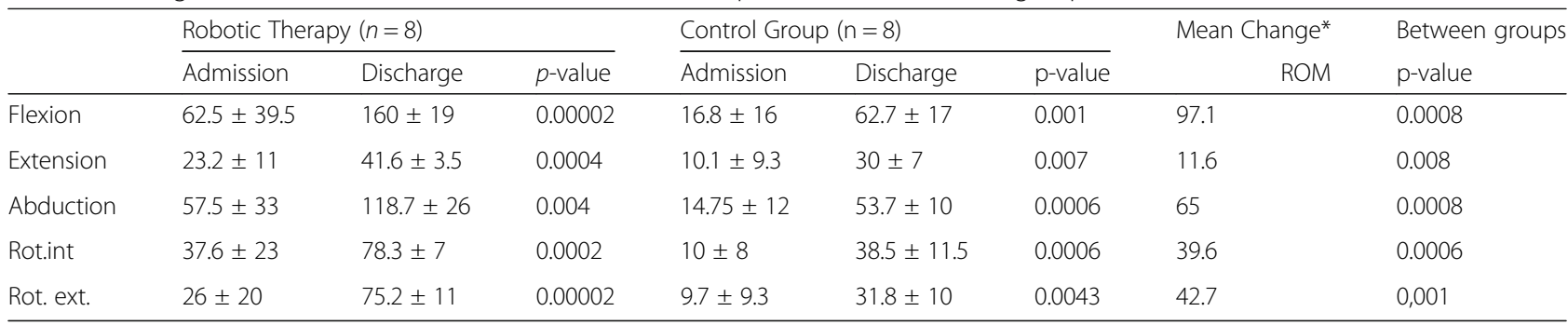

Rot.int. - Internal rotation.

Rot.ext. -External rotation.

$\left.{ }^{*}\right)$ Mean difference between discharges of both groups.

in flexion shoulder. However the rest of the patients of this experimental group presented high degrees of functional disability.

The increase in joint mobility experienced by patients in the robotic therapy group significantly exceeded those achieved by patients in the control group, in which only one of the patients meets the functionality criteria established in this work. The continuous and significant gradual increase in active joint movement (ROM) of these patients from the admission is the result largely of the control and reduction of spasticity that limits, as it is known the active flexion, abduction and external rotation patterns and the consequent early elimination of pain. These aspects, which however have little or no change during conventional therapy delayed pain elimination, that take place in a period of time that undermines the prediction for better rehabilitation rates [33, 34-36].

From Fig. 3, it is deductible that both rehabilitative treatments were effective. From Table 7 it can be observed that ROM improvements were statistically significant inside both groups, as well as the difference between them, for each of the therapeutic movements involved. However, it should be noted that despite its better ROM indicators during admission, in the functional recovery of the shoulder, the robotic therapy group showed greater increases in the range of joint mobility at the end of the treatment.

Table 7 shows the mean difference during the discharge between both groups for the different movements involved. As noted, such a difference between both treatments is very significant. Despite heterogeneity in the experimental group, robotic therapy achieved an average increase of 97 degrees in shoulder flexion, 65 degrees in abduction and about 40 degrees in rotations, far away from the improvements provided by the conventional therapy.

In this regard, it is worth highlighting those cases of the experimental group whose indicators during admission were comparable to those of the conventional group and nonetheless reach recovery rates on par with the rest of the experimental group, for example the patient with a flaccid limb and the chronic patient S5, who
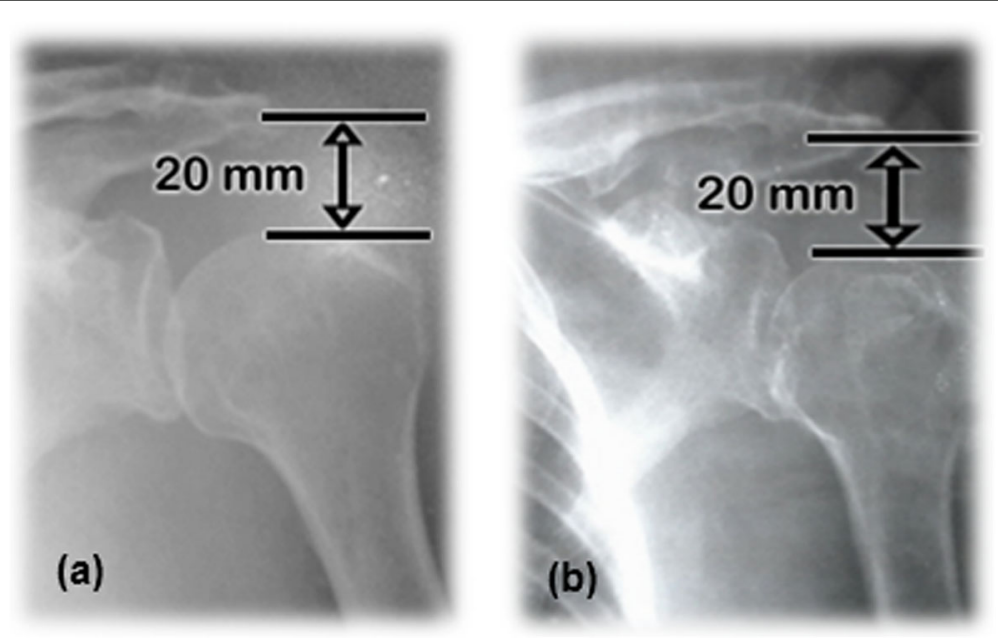

Fig. 4 HSS. (a) The patient C3 from control group during admission. (b) The patient C3 from control group after 3 months of conventional therapy 

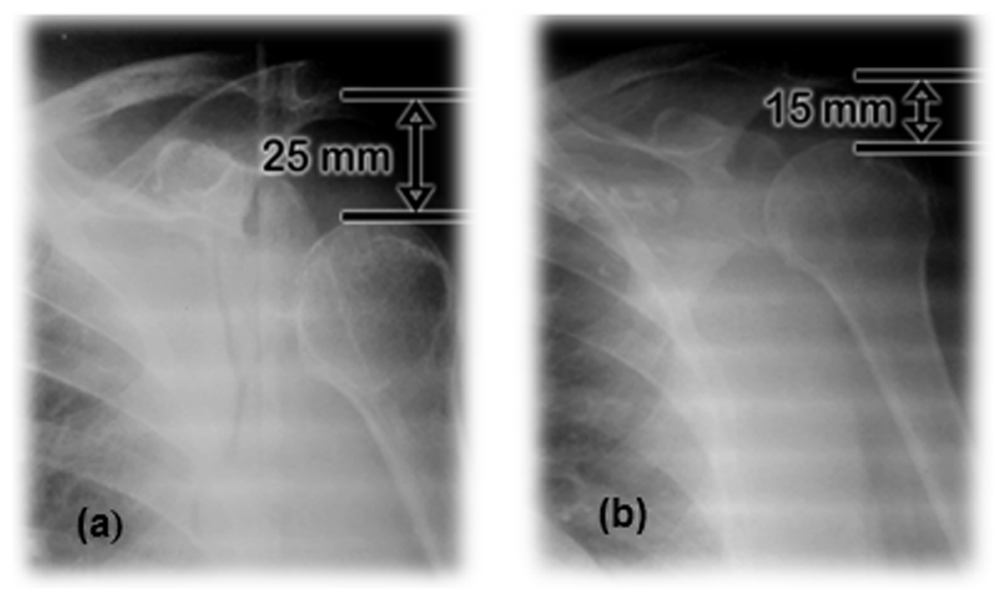

Fig. 5 HSS. a The patient from robotic therapy group during admission. b The patient from experimental group after 3 months of robotic therapy

receiving prior conventional therapy entered the experimental group with severe subluxation, spasticity, even with pain and very discrete degrees of joint mobility.

This effect could be explained in how the decrease in spasticity and pain allow a gradual increase in the arcs of articular mobility of the shoulder in patients from the experimental group.

Another important outcome is referred to the recovery of motor capacity expressed in the evaluation of muscle strength from admission and during treatment. From Tables 3 and 5 it is deduced that conventional therapy after 3 months of treatment, although it experienced changes with statistically significance $(p<0.002)$ in muscle tone patients did not show an important recovery as robotic therapy (Tables 4 and 6 ). There was statistically difference between groups $(p<0.0014)$.

It can be concluded that robotic therapy in general prompted an increase in muscle strength in the patients (according to the reported results in the MRC scale). Only patient S5, who showed since admission to 3 months a value of muscular strength $\mathrm{MRC}=2$ was the exception, coincidentally being the patient with an evolution time of 14 months and a great subluxation in the left arm, aspects that seem to have influenced in the recovery of the muscle tone.

In general, a greater muscular strength can be observed associated with those patients who achieve greater active ranges of joint movement with robotic therapy, as it has been suggested by other authors $[10$, $32,34,35,37]$.

Considering the nature of such pathologies, it is noteworthy the motivation and enthusiasm of the patients during the treatment with robotic therapy. A great satisfaction degree of the people being treated (Table 6), constitutes an indicator not only from the patient's satisfaction with the result of the therapy, but also constitutes an aspect that positively influences the process of neurorrehabilitación of the hemiplegic patient with the use of exoskeletons.

Finally, all the functional and motor achievements reached by the patients from experimental group were sustained over time, showing in this way, the potential of such treatment in the early recovery of the hemiplegic shoulder.

Although it was not the object of this study, significant results were reported in the rehabilitation of the different indicators in the elbow joint and to a lesser extent of the wrist [38], in addition to very positive impacts on the concentration and the physical capacity of the patients.

\section{Conclusions}

The outcomes of this study favored robotic therapy over conventional therapy in an early elimination of pain in a significant way $(20 \div 30$ sessions), which enables the obtaining of better rehabilitation indicators. It means that it is possible to control pain through reducing shoulder spasticity using a proper upper limb exoskeleton therapy.

As it has been reported in the scarce literature on shoulder subluxation in the hemiplegic patient, a rapid and significant decrease in the magnitude of subluxation even in chronic patients (evolution times greater than 6 months) was obtained. In this sense, it is worth mentioning the attainment rehabilitation indices in patients with different evolution times for different etiologies of the painful shoulder of the hemiplegic patient.

Our findings and outcome results were adjusted for the data for this study, even when small sample size was used. A large number of patients would allow more conclusive results than those presented here. 
These findings suggest that the lack of mobility that follows the stroke is the main cause of the development of spasticity, muscle contractures, flaccidity and subluxations as multifactorial causes of shoulder pain. Indeed the performance of precise routines and gradually controlled movements, lead to the decrease of these abnormal muscle tones, the disappearance of pain and the greater recovery of joint movement with the use of robotic therapy.

\begin{abstract}
Abbreviations
WHO: World Health Organization; CVA: Cerebrovascular accidents: HSP: Hemiplegic shoulder pain; MAS: Modified Ashworth scale; MRC: Scale of the Medical Research Council; HSS: Humeral scapular subluxation; SHSS: Without subluxation (neurological etiology or other osteomioarticular causes); BCl: Brain-computer interface; BMI: Brain Machine Interface; tDCs: Transcranial direct current stimulation; DOF: Degrees of freedom; ESS: Evaluation of shoulder stability; AJM: Functionality degree of active joint movement; ROM: Range of joint mobility
\end{abstract}

\section{Acknowledgements}

The authors wish to acknowledge the collaboration and the financial support of the University Corporation of Hulia, Colombia and the crew of Rehabilitation Department at Surgical Clinical Hospital "Dr. Juan Bruno Zayas" from Santiago de Cuba, Cuba. Finally, we would like to thank all patients who kindly participated in this study.

\section{Authors' contributions}

RSZ participated to conception, coordination and implementation of the whole research project. MTQ, DMH, RSR and RSZ developed the robotics system used in the study. MHZ, AMP and RSZ performed the daily therapy and control test over the course of the clinical pilot study. MHZ and AMP analyzed the data; RSZ and MHZ wrote the manuscript. All authors read and approved the final manuscript.

\section{Funding}

The development of the robotic exoskeleton was financed by the Universidad de Oriente of Santiago de Cuba, Cuba with the collaboration of the University Corporation of Huila. All the expenses incurred in the realization and processing of the results of the pilot study were assumed by the "Juan Bruno Zayas" Surgical Clinical Hospital. The University Corporation of Huila assumes the expenses derived from the writing, translation and publication of this research report.

\section{Availability of data and materials}

The datasets used and analyzed during the current study are registered in the final reports from the research project "Robotic therapy for the treatment of the painful hemiplegic shoulder" at the surgical clinical Hospital "Dr. Juan Bruno Zayas" and PhD thesis of Mauricio Torres Quezada at the University of Oriente repository and are available on reasonable request.

\section{Ethics approval and consent to participate}

The research has been carried out in accordance with the ethical principles for human research established in the Declaration of Helsinki. All subjects signed informed consent documents include the respect for privacy and their right to leave the research once they decided so, without prejudice to the continuity of their medical care. The study was approved by the institutional Ethics and Scientific Committees at the Surgical Clinical Hospital "Dr. Juan Bruno Zayas".

\section{Consent for publication}

All patients were informed about the purpose of the investigation, characteristics and procedure, which would not cause any harm to health, its possible benefits and results in the functional rehabilitation of their condition. They were asked for their informed consent for inclusion in the study, as well as the publication and dissemination of the results, signed, dated and approved.

\section{Competing interests}

The authors have no competing interests in relation to this research.

\section{Author details}

${ }^{1}$ University Corporation of Hulia, Nevia, Huila, Colombia. ${ }^{2}$ Universidad de Oriente, Santiago de Cuba, Cuba. ${ }^{3}$ Surgical Clinical Hospital "Juan Bruno Zayas", Santiago de Cuba, Cuba.

Received: 1 April 2019 Accepted: 1 April 2020

Published online: 22 April 2020

\section{References}

1. Anuario estadistico de salud año 2014. Dirección de estadisticas y regristros médicos. Ministerio de Salud Pública de la República de Cuba La habana. 2015; ISSN: 1561-4425.

2. Lombillo Laferté LM, Martínez Segón S, Serra Valdés Y, Rodríguez Mutuberría L. Complicaciones en pacientes hemipléjicos por ictus. Rev Cubana de Medicina. 2014;53(2):134-43.

3. Madera $P$, Isabel C. Epidemiología de las enfermedades cerebrovasculares de origen extra craneal. Revista cubana de Angiol Circ Vas. 2014;15(2):74-83.

4. Hanoi SH, Liliana BV, Silvia TS. Rehabilitación del ictus. Primera parte. Revista Cubana de Medicina Física y Rehabilitación. 2015;7(1):84-95.

5. Pons J. L. and Torricelli D. (eds.), Emerging Therapies in Neurorehabilitation, Biosystems \& Biorobotics 4, DOI: https://doi.org/10.1007/978-3-642-38556-8_10, (c) Springer-Verlag Berlin Heidelberg 2014.

6. Chen CC, Bode RK. Factors influencing therapists' decision-making in the acceptance of new technology devices in stroke rehabilitation. Am J Phys Med Rehabil. 2011;90(5):415-25.

7. Brewer L, Horgan F, Hickey A, Williams D. Stroke rehabilitation: recent advances and future therapies. QJM. 2012.

8. Dohle Carolin I, et al. Pilot study of a robotic protocol to treat shoulder subluxation in patients with chronic stroke. J NeuroEng Rehabil. 2013;10:88.

9. Pedrocchi A, et al. MUNDUS project: multimodal Neuroprosthesis for daily upper limb Suppor. J NeuroEng Rehabil. 2013;10:66.

10. Patricia S, et al. Effects of intensive arm training with the rehabilitation robot ARMin II in chronic stroke patients: four single cases. J NeuroEng Rehabil. 2009;6:46.

11. Frisoli, Antonio et al. Training and assessment of upper limb motor function with a robotic exoskeleton after stroke. The Fourth IEEE RAS/EMBS International Conference on Biomedical Robotics and Biomechatronics Roma, Italy. June 24-27, 2012.

12. Abdullah HA, et al. Results of clinicians using a therapeutic robotic system in an inpatient stroke rehabilitation unit. J NeuroEng Rehabil. 2011;8:50.

13. Min-Su Kim et al Robotic-assisted shoulder rehabilitation therapy effectively improved Poststroke hemiplegic shoulder pain: a randomized controlled trial. Arch Phys Med Rehabil 2019. 100(6) 1015-1022 https://doi.org/10.1016/ j.apmr.2019.02.003.

14. Kwakkel G. Effects of robot-assisted therapy on upper limb recovery after stroke: a systematic review. Neurorehabil Neural Repair. 2008;22(2):111-21. https://doi.org/10.1177/1545968307305457.

15. Veerbeek JM. Effects of robot-assisted therapy for the upper limb after Stroke: A systematic review and meta-analysis. Neurorehabil Neural Repair. 2017;31(2):107-21.

16. Coscia $M$, et al. Neurotechnology-aided interventions for upper limb motor rehabilitation in severe chronic stroke. Brain. 2019;142:2182-97.

17. Torres Quezada, M.; Sagaró Zamora, R.; Broche Vázquez, L.; Delisle Rodríguez, D., and López Delis, A. Robotic Training System for Upper Limb Rehabilitation. Ingeniería y Universidad. 2014, vol. 18, no. 2, pp. 235-252. http://dx.doi. org/https://doi.org/10.11144/Javeriana.IYU18-2.rtsu.

18. Torres Quezada, M.; Sagaró Zamora, R.; Broche Vázquez, L.; Delisle Rodríguez, D.; Reyes Ángel, López Delis a.; Rossi Esteban. Robotic system for upper limb rehabilitation. IFMBE proceedings (International Federation for Medical and Biological Engineering, springer Verlag). Volume 49, 2015, pp 948-951. DOl:https://doi.org/10.1007/978-3-319-13117-7_240. Springer International Publishing ISBN 978-3-319-13117-7.

19. M. Kosaka et al (eds.). Thermotherapy for Neoplasia, Inflammation, and Pain (c) Springer Japan 2001.

20. I. Cifu, David X. (ed). Braddom's physical medicine and rehabilitation. Fifth edition. ISBN: 978-0-323-28046-4. Elsevier, Inc. 2016.

21. Pavez Ulloa FJ. Agentes físicos superficiales y dolor. Análisis de su eficacia a la luz de la evidencia científica. Rev Soc Esp Dolor. 2009;16(3):182-9. 
22. Dong Huang et al Effects of Linear-Polarized Near- Infrared Light Irradiation on Chronic Pain. The Scientific World Journal Volume 2012, Article ID 567496, 4 pages doi:https://doi.org/10.1100/2012/567496.

23. Walsh K. Management of shoulder pain in patients with stroke. Postgrad Med J. 2001;77:645-9. https://doi.org/10.1136/pmj.77.912.645.

24. Murie-Fernández M, Iragui C, Gnanakumar V, Meyer M, Foley N, Teasell R. Hombro doloroso hemipléjico en pacientes con ictus, causas y manejo. Neurología. 2012;27(4):234-44.

25. Vuagnat $\mathrm{H}$, Chantraine A. Shoulder pain in hemiplegia revisited: contribution of functional electrical stimulation and other therapies. J Rehabil Med. 2003;35:49-54.

26. Meskers CG, Koppe PA, Konijnenbelt MH, Veeger DH, Janssen TW. Kinematic alterations in the ipsilateral shoulder of patients with hemiplegia due to stroke. Am J Phys Med Rehabil. 2005;84:97-105.

27. Hecht JS. The role of spasticity in hemiplejic shoulder pain and what to do about it. 57th annual assembly of American Academy of Ohysical medicine and. Rehabilitation. 1995:248-55.

28. Bender L, McKenna K. Hemiplegic shoulder pain: defining the problem and its management. Disabil Rehabil. 2001;23:698-705.

29. Gilmore PE, Spaulding SJ, Vandervoort AA. Hemiplegic shoulder pain: implications for occupational therapy treatment. Can J Occup Ther. 2004;71: 36-46.

30. Mirallas Martínez JA. Avances en la rehabilitación del paciente con enfermedad cerebrovascular. Rehabilitación (Madrid). 2004;38(2):78-85

31. Enrique AM, et al. Resultados del tratamiento fisioterapéutico mediato en pacientes con enfermedad cerebrovascular. Revista Cubana de Medicina General Integral. 2010;26(4):617-23.

32. Ellis MD, Holubar BG, Acosta AM, Beer RF, Dewald JP. Modifiability of abnormal isometric elbow and shoulder joint torque coupling after stroke. Muscle Nerve. 2005;32:170-8.

33. Palazón García R, et al. Hombro doloroso en el hemipléjico. Rehabilitación (Madr). 2004;38(3):104-7.

34. Ramos Valero L. Tratamientos fisioterápicos para pacientes con hombro doloroso tras un accidente cerebrovascular. Fisioterapia. 2013; 35(5):214-23.

35. Frolov, A. A et al. Use of Robotic Devices in Post-Stroke Rehabilitation. Neuroscience and Behavioral Physiology, Vol. 48, No. 9, 2018.

36. Qiuyang Qian et al Distal versus proximal - an investigation on different supportive strategies by robots for upper limb rehabilitation after stroke: a randomized controlled trial. Journal of NeuroEngineering and Rehabilitation (2019) 16:64, doi.org/10.1186/s12984-019-0537-5.

37. Ansari NN, et al. Efficacy of therapeutic ultrasound and infrared in the management of muscle spasticity. Brain Injury. 2009;23(7-8):632-8.

38. Van Ouwenaller C, Laplace P, Chantraine A. Painful shoulder in hemiplegia. Arch Phys Med Rehabil. 1986;46:23-6.

\section{Publisher's Note}

Springer Nature remains neutral with regard to jurisdictional claims in published maps and institutional affiliations.

Ready to submit your research? Choose BMC and benefit from:

- fast, convenient online submission

- thorough peer review by experienced researchers in your field

- rapid publication on acceptance

- support for research data, including large and complex data types

- gold Open Access which fosters wider collaboration and increased citations

- maximum visibility for your research: over $100 \mathrm{M}$ website views per year

At $\mathrm{BMC}$, research is always in progress.

Learn more biomedcentral.com/submissions 